\title{
A likely optical counterpart of the G292.0+1.8 pulsar wind nebula ${ }^{\star}$
}

\author{
S. V. Zharikov ${ }^{1}$, Yu. A. Shibanov ${ }^{2}$, D. A. Zyuzin ${ }^{2,3}$, R. E. Mennickent ${ }^{4}$, and V. N. Komarova ${ }^{5}$ \\ 1 Observatorio Astronómico Nacional SPM, Instituto de Astronomía, Universidad Nacional Autónomia de Mexico, Ensenada, BC, \\ Mexico \\ e-mail: zhar@astrosen.unam.mx \\ 2 Ioffe Physical Technical Institute, Politekhnicheskaya 26, St. Petersburg 194021, Russia \\ e-mail: shib@astro.ioffe.ru \\ 3 Academical Physical Technical University, Khlopina 8, St. Petersburg 195220, Russia \\ 4 Departamento de Fisica, Universidad de Concepcion, Casilla 160-C, Concepcion, Chile \\ e-mail: rmennick@astro-udec.cl \\ 5 Special Astrophysical Observatory Russian Academy of Science, Nizhnii Arkhyz, Russia \\ e-mail: vkom@sao.ru
}

Received 19 June 2008 / Accepted 10 October 2008

\section{ABSTRACT}

\begin{abstract}
Context. G292.0+1.8 is a Cas A-like supernova remnant containing the young pulsar PSR J1124-5916, which powers a compact torus-like pulsar wind nebula with a jet visible in X-rays.

Aims. We have performed deep optical observations of the pulsar field to detect the optical counterpart of the pulsar and its nebula. Methods. The observations were carried out using the direct imaging mode of FORS2 at the ESO VLT/UT1 telescope in the $V, R$, and $I$ bands. We also analyzed archival images obtained with the Chandra/ACIS-I, ACIS-S, and HRC-S in X-rays.

Results. In all three optical bands we detect a faint elliptical nebulosity whose brightness peak and center position are consistent at a sub-arcsecond level with the X-ray position of the pulsar. The field is densely packed with background stars, but after subtraction of these stars the morphology of the object and the orientation of its major axis appear to be in good agreement with the brightest inner part of the pulsar nebula torus region seen almost edge on in X-rays. Within the nebulosity we do not resolve any point-like optical object that could be identified with the pulsar and estimate its contribution to the observed nebulosity flux as $\$ 20 \%$. Extracting the $\mathrm{X}$-ray spectrum from the physical region equivalent to the optical source position and extent and combining that with the measured optical fluxes, we compile a tentative multi-wavelength spectrum of the inner part of the nebula. Within uncertainties of the interstellar extinction towards G292.0+1.8 it is reminiscent of either the Crab or PSR B540-69 and J0205+6449 pulsar wind nebula spectra. Conclusions. The position, morphology, and spectral properties of the detected nebulosity suggest that it is the likely optical counterpart of the pulsar plus its wind nebula system in G292.0+1.8. Higher spatial resolution optical observations and the extension of the broad-band spectrum of the proposed counterpart candidate towards the IR and UV are necessary to confirm its origin.
\end{abstract}

Key words. stars: pulsars: general - stars: pulsars: individual: G292.0+1.8 - stars: neutron

\section{Introduction}

Optical emission from radio pulsars contains important information on the poorly understood physical processes responsible for the multi-wavelength radiation of rotation-powered neutron stars (NSs). So far only a small part $(\leq 1 \%)$ of the $\sim 1700$ known radio pulsars has been detected in the optical range (Mignani 2005). The most luminous of them are relatively young and energetic and produce compact torus-like pulsar wind nebulae (PWNe), which are typically visible in X-rays. All these young optical pulsars are associated with supernova remnants (SNRs): Crab, PSR B0540-69 (SNR B0540-69.3 in the LMC), PSR B150958 (G320.4-1.2), and Vela. Only for the first two of these pulsars has the surrounding PWN also been identified in the optical range (e.g., Serafimovich et al. 2004). The multi-wavelength data of the Crab pulsar and its PWN show the power-law spectra

\footnotetext{
* Based on observations made with ESO telescope at the Paranal Observatory under Programme 78.D-0617(A) and with archival ESO VLT data, obtained from the ESO/ST-ECF Science Archive Facility.
}

with one spectral break between the optical and X-rays, while the spectral energy distribution for PSR B0540-69 and its PWN suggest a double-knee break in the same range. Recent optical (Shibanov et al. 2008) and mid-infrared (Slane et al. 2008) detections of the torus-like PWN around PSR J0205+6449 in the Crab-like SNR 3C 58 perhaps also suggest the double-knee break. Additional optical identifications can provide new insights into this issue.

The young PSR J1124-5916 only recently has been discovered in the radio (Camilo et al. 2002) and X-rays (Hughes et al. 2001, 2003). It is associated with the SNR G292.0+1.8 (MSH 11-54) which is the third oxygen-rich SNR known in the Galaxy after Cas A and Pupis A. G292.0+1.8 has a morphology similar to Cas A on small scales (Hwang et al. 2000); however, no Fe $\mathrm{K}$ line emission was detected in its spectrum. Like the Crab pulsar, PSR J1124-5916 powers a torus-like X-ray PWN with a jet (Hughes et al. 2001; Safi-Harb \& Gonzalez 2002; Hughes et al. 2003; Park et al. 2007). The pulsar period, $135 \mathrm{~ms}$, and the period derivative, $7.4 \times 10^{-13}$, imply a characteristic age of 

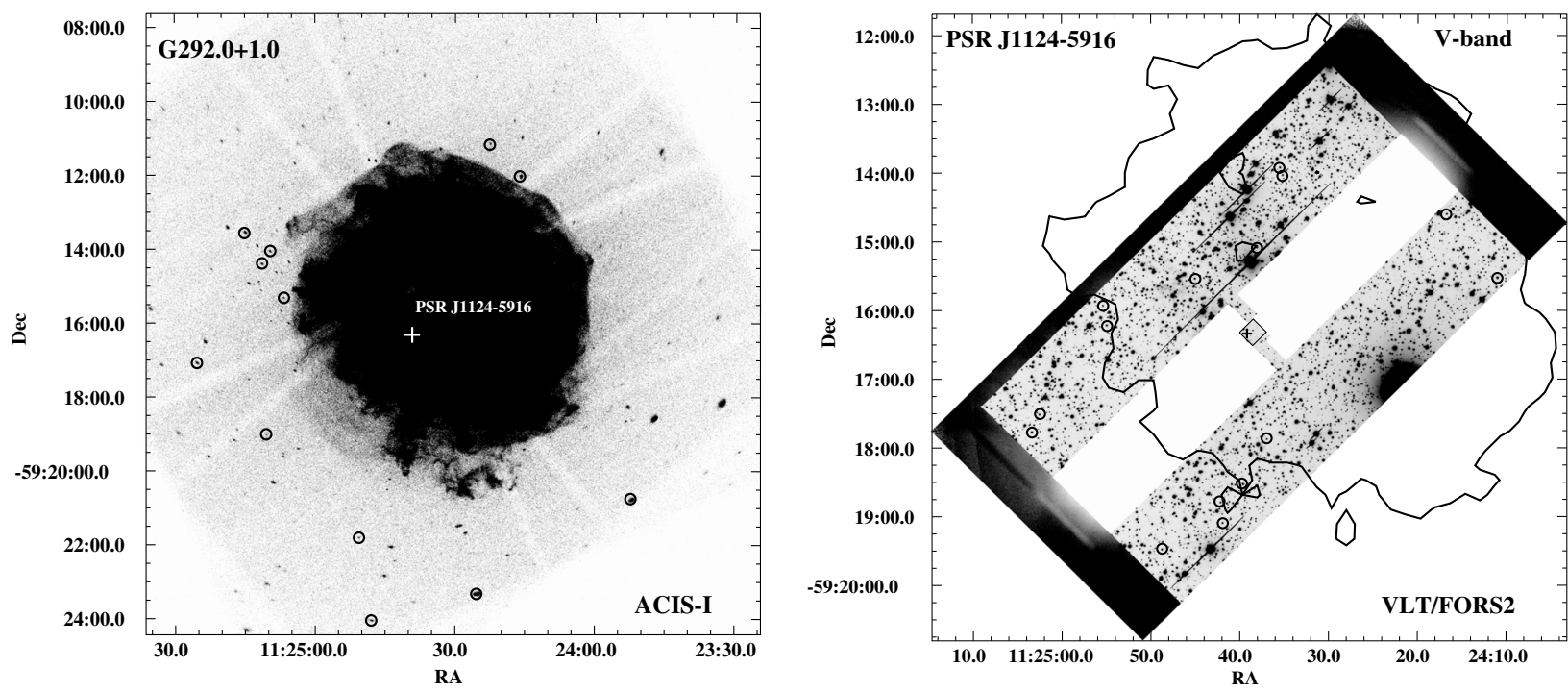

Fig. 1. The optical VLT/V-band (left) and the X-ray Chandra/ACIS-I (right) images of the PSR J1124-5916/SNR G292.0+1.8 field. White rectangular regions on the VLT CCD chip are covered by occulting bars to eliminate the contamination in the pulsar neighborhood by the light from bright field stars. An outermost SNR contour from the X-ray image is overlaid on the optical image. Circles mark the USNO stars used for astrometric referencing of the images, the cross marks the pulsar position. The small box in the center of the VLT/V-band image is enlarged in Fig. 2.

$\tau \approx 2900 \mathrm{yr}$, consistent with a 2700-3700 yr age of the SNR (Camilo et al. 2002; Chevalier 2005). The derived spin-down luminosity of this pulsar is $\dot{E}=1.2 \times 10^{37} \mathrm{erg} \mathrm{s}^{-1}$. This ranks PSR J1124-5916 as the sixth youngest and the eighth most energetic among the all rotation-powered pulsars known. Because of its age, optical properties, and association with the pulsar and PWN, G292.0+1.8 most closely resembles the oxygen-rich SNR 0540-69.3 in the LMC. At the same time, the spin-down energy loss of PSR J1124-5916 makes this pulsar closer to the older Vela-pulsar which is known to be very under-luminous in the optical and X-rays.

The discovery of PSR J1124-5916 and its PWN in X-rays has prompted deeper narrow-band and spectral optical studies of G292.0+1.8 (Winkler \& Long 2006) as well as broad-band searches for the pulsar optical counterpart (Hughes et al. 2004). Nevertheless, no counterpart has been detected with the CTIO $4 \mathrm{~m}$ telescope down to an estimated stellar visual limit of $\sim 26.4$ mag. Assuming a distance of $d \approx 6 \mathrm{kpc}$ and an interstellar reddening toward the pulsar of $A_{V} \approx 1.8-2.2 \mathrm{mag}$, this implies that the intrinsic optical luminosity for PSR J1124-5916 must be lower than that of the younger Crab, B0540-69, and B1509-58 pulsars, but still above the older Vela pulsar. This is also supported by the comparison of the X-ray luminosity of this pulsar with an empirical relation between the optical and $\mathrm{X}$-ray luminosities of the pulsars detected in both spectral domains (Zharikov et al. 2002, 2004, 2006; Zavlin \& Pavlov 2004), suggesting further optical studies of the PSR J1124-5916 field.

Here we report new deep optical imaging of the PSR J11245916 field in the $V, R$ and $I$ bands with the ESO Very Large Telescope (VLT). The observations allowed us to find a likely candidate optical counterpart of the J1124-5916 pulsar+PWN system. We compare our optical images with the X-ray data retrieved from the Chandra archive ${ }^{1}$. The observations and data reduction are described in Sect. 2. We present our results in Sect. 3 and discuss them in Sect. 4.

\footnotetext{
1 ACIS-I, Obs 6677-6680, 8221, 8447, 530 ks exposure, PI Park.
}

Table 1. Log of the VLT/FORS2 observations of PSR J1124-5916.

\begin{tabular}{llccl}
\hline \hline Date & $\begin{array}{l}\text { Band, dithered } \\
\text { Exposure number }\end{array}$ & $\begin{array}{c}\text { Exposure } \\
\text { length, } s\end{array}$ & Airmass & $\begin{array}{l}\text { Seeing } \\
\text { arcsec }\end{array}$ \\
\hline $2007-01-26$ & $V, 10$ & 595 & 1.22 & 0.6 \\
$2007-01-27$ & $V, 5$ & 595 & 1.22 & 0.6 \\
& $I, 7$ & 200 & 1.24 & 0.6 \\
$2007-02-20$ & $R, 12$ & 595 & 1.24 & 0.8 \\
& $I, 4$ & 200 & 1.29 & 0.85 \\
\hline
\end{tabular}

\section{Observations and data reduction}

\subsection{Observations}

The images of the pulsar field were obtained in the Bessel $V, R$ and $I$ bands with the FOcal Reducer and low dispersion Spectrograph (FORS2 ${ }^{2}$ ) at the VLT/UT1 (ANTU) unit during several service mode runs in January and February 2007. A standard resolution mode was used with an image scale of $\sim 0$.'251/pixel and a field of view (FOV) of $\sim 7^{\prime} \times 7^{\prime}$. Because G292.0+1.8 and its pulsar are located near the Galactic plane, which is densely packed with stars, we used a FORS2 occultingbar setup for our observations. This allowed us to minimize contamination over the pulsar region by illumination and saturation spikes from bright field stars surrounding the pulsar (see Fig. 1, left panel). Sets of 3-10 min. dithered exposures were obtained in each of the bands with a total integration time of $8925 \mathrm{~s}$, $7140 \mathrm{~s}$, and $2200 \mathrm{~s}$ in the $V, R$ and $I$ bands. The observing conditions were rather stable with seeing values varying from 0.6 to $0 . ' 85$. The $\log$ of the observations is given in Table 1. Standard data reduction including bias subtraction, flat-fielding, and cosmic ray trace removing was performed making use of the IRAF and MIDAS tools.

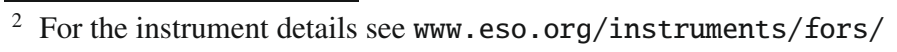




\subsection{Astrometric referencing}

Astrometric referencing was applied to the summed VLT images in each of the bands. To obtain a precise astrometric solution, the positions of the astrometric standards selected from the USNO-B1 astrometric catalog ${ }^{3}$ were used as a reference. Thousands of USNO-B1 reference objects can be identified in our FOV, which is extremely crowded by stellar objects. The recent release of the Guide Star Catalog (GSC-II v2.3.2) ${ }^{4}$ provides practically the same number of the standards but contains no information on proper motions and the declared astrometric errors $(0.3)$ are higher than the nominal 0.2 uncertainty of the USNO-B1. A number of stars from the UCAC2 catalog is also present but they are saturated in our images. We discarded the reference stars with significant proper motions and catalog positional uncertainties $\gtrsim 0$ '. 4 along with the saturated ones. Finally, to minimize potential positional uncertainties caused by overlapping stellar profiles in the crowded FOV, we selected only 15 isolated stars (see Table 2 and Fig. 1, left panel). Their pixel coordinates were derived making use of the IRAF task imcenter. The IRAF tasks ccmap/cctran were applied for the astrometric transformation of the images. Formal rms uncertainties of the astrometric fit for our images are $\Delta \mathrm{RA} \lesssim 00^{\prime} 07$ and $\Delta \mathrm{Dec} \lesssim 00^{\prime} 05$ for each of the bands, and the fit residuals are $\$ 0$.' 13 , which is compatibile with the maximal catalog position uncertainty of the selected standards. Selection of another set of isolated reference objects does not significantly change the result and a conservative estimate of our $1 \sigma$ astrometric referencing uncertainty is $\lesssim 0$ ' 15 in both RA and Dec for all three optical bands.

For further comparison of the optical and X-ray images we have also performed astrometric referencing of the G292.0+1.8 Chandra/ACIS-I archival X-ray images. We combined the multiple data sets obtained with the ACIS-I making use of the merge_all v3.6 script of the CIAO tool. The resulting image allowed us to resolve tens of point-like objects located outside the SNR X-ray boundary (Fig. 1, right panel). Most of them can be identified with the USNO standards. Potential reference objects within the SNR were ignored to exclude any false identification with numerous X-ray knots and filaments within the SN remnant. Using similar selection criteria as in the optical case, we finally selected 12 suitable standards, listed in Table 2 and marked in Fig. 1 (right panel). The resulting rms uncertainties of the respective astrometric fit are $\Delta \mathrm{RA} \approx 0.187$ and $\Delta \mathrm{Dec} \approx 0 . ' 122$ with the fit residuals of $\lesssim 0 \prime^{\prime} 27$. This is compatible with the maximal positional uncertainty of the selected standards and makes the quality of the astrometric referencing of the ACIS-I image comparable to that of the VLT images. The resulting shift between the initial and transformed X-ray images was $\approx 0.4$ of the ACIS pixel size $(0.5)$ or about 0.22 mainly directed towards the east. This ensures an almost perfect pointing accuracy of the X-ray observations of the G292.0+1.8 field with the ACIS-I.

\subsection{Photometric calibration}

The observing conditions during our observations were photometric. The photometric calibration was carried out with PG 1323-086, PG 1633+099, PG 0231+051, and RUBIN149 photometric standards from Landolt (1992) observed during the

\footnotetext{
3 USNO-B1 is currently incorporated into the Naval Observatory Merged Astrometric Data-set (NOMAD) which combines astrometric and photometric information from Hipparcos, Tycho-2, UCAC, Yellow-Blue6, USNO-B, and the 2MASS, wWw . nofs . navy.mil/data/fchpix/

4 www-gsss.stsci.edu/Catalogs/GSC/GSC2/GSC2 . htm
}

Table 2. USNO-B1.0 stars used for astrometrical referencing with coordinates (epoch $\mathbf{J} 2000.0$ ) and their $1 \sigma$ errors.

\begin{tabular}{|c|c|c|c|c|}
\hline$\overline{\text { Name }}$ & $\begin{array}{c}\text { RA } \\
\text { hh mm ss }\end{array}$ & $\begin{array}{c}\text { Dec } \\
\text { dd mm ss }\end{array}$ & $\begin{array}{l}\sigma_{\mathrm{RA}} \\
\mathrm{mas}\end{array}$ & $\begin{array}{l}\sigma_{\mathrm{Dec}} \\
\mathrm{mas}\end{array}$ \\
\hline & VLT & images & & \\
\hline $0307-0263383$ & 112435.5253 & -591355.840 & 18 & 23 \\
\hline $0307-0262730$ & 112416.8207 & -591436.360 & 92 & 79 \\
\hline $0307-0262544$ & 112411.1133 & -591531.800 & 10 & 39 \\
\hline 0306-0262602 & 112448.7647 & -591928.770 & 75 & 23 \\
\hline 0307-0263370 & 112435.2033 & -591402.500 & 44 & 163 \\
\hline $0307-0263484$ & 112438.1093 & -591505.450 & 44 & 54 \\
\hline 0307-0263768 & 112445.0667 & -591532.820 & 92 & 228 \\
\hline 0307-0263438 & 112436.9907 & -591752.570 & 9 & 77 \\
\hline 0306-0262194 & 112439.6380 & -591831.850 & 63 & 40 \\
\hline 0306-0262311 & 112442.1680 & -591847.830 & 148 & 36 \\
\hline 0306-0262297 & 112441.9627 & -591905.730 & 56 & 106 \\
\hline 0307-0264210 & 112454.9427 & -591613.580 & 40 & 37 \\
\hline 0307-0264219 & 112455.3613 & -591556.250 & 138 & 40 \\
\hline 0307-0264485 & 112502.5093 & -591730.950 & 122 & 100 \\
\hline \multirow[t]{2}{*}{$0307-0264529$} & 112503.3600 & -591746.450 & 50 & 79 \\
\hline & Chandra/ACIS-I & image & & \\
\hline 0307-0294705 & 112506.7700 & $-59: 15: 19.710$ & 171 & 74 \\
\hline 0307-0292657 & 112416.0510 & $-59: 12: 02.614$ & 12 & 12 \\
\hline 0306-0287673 & 112425.3659 & $-59: 23: 19.419$ & 43 & 56 \\
\hline 0306-0286344 & 112352.2471 & $-59: 20: 46.686$ & 15 & 15 \\
\hline 0308-0292285 & 112422.6380 & $-59: 11: 09.990$ & 32 & 59 \\
\hline 0307-0294814 & 112509.6299 & -591404.161 & 0 & 0 \\
\hline 0307-0295391 & 112525.2547 & -591704.560 & 200 & 200 \\
\hline 0305-0283993 & 112447.9537 & -592402.740 & 0 & 0 \\
\hline 0306-0288792 & 112450.6720 & -592148.220 & 122 & 50 \\
\hline 0306-0289663 & 112510.4193 & -591901.500 & 50 & 184 \\
\hline 0307-0294870 & 112511.3865 & -591424.310 & 0 & 0 \\
\hline 0307-0295000 & 112514.9267 & -591333.540 & 313 & 61 \\
\hline
\end{tabular}

same nights as our target. We fixed the atmospheric extinction coefficients at their mean values adopted from the VLT home page $^{5}: k_{V}=0.11 \pm 0.01, k_{R}=0.03 \pm 0.01$, and $k_{I}=0.00 \pm$ 0.01. The first observing run on 2007-01-26, when the standards were observed at significantly different airmasses, allowed us to check that the atmospheric extinction during our observations was really in agreement with the mean one. The photometric fits performed for each of the observing nights showed that the photometric zero-points did not vary significantly from night to night despite day and even month gaps in the integration times for the $V$ and $I$ bands (Table 1). As a result, we obtained the following magnitude zero-points for the summed images; $V^{\mathrm{ZP}}=27^{\mathrm{m}} .83 \pm 0.02, R^{\mathrm{ZP}}=27^{\mathrm{m}} .95 \pm 0.02$, and $I^{\mathrm{ZP}}=27^{\mathrm{m}} .31 \pm$ 0.02 , where the errors account for the statistical uncertainties of magnitude measurements, the extinction coefficient uncertainties, and marginal zero-point variations from night to night. The formal $3 \sigma$ detection limits of a point-like object in the co-added images for one-arcsecond aperture are $V \approx 27.8, R \approx 26.9$, and $I \approx 25.7$.

\section{Results}

\subsection{Detection of the pulsar/PWN counterpart candidate}

The region containing the pulsar is enlarged in Fig. 2, where we compare our optical $V, R$, and $I$ images with the archival $\mathrm{X}$-ray images obtained with Chandra/ACIS-I and HRC-S ${ }^{6}$. The

\footnotetext{
5 http://wwW . eso.org

6 HRC-S, Obs 1953, 49.8 ks exposure, PI Hughes.
} 

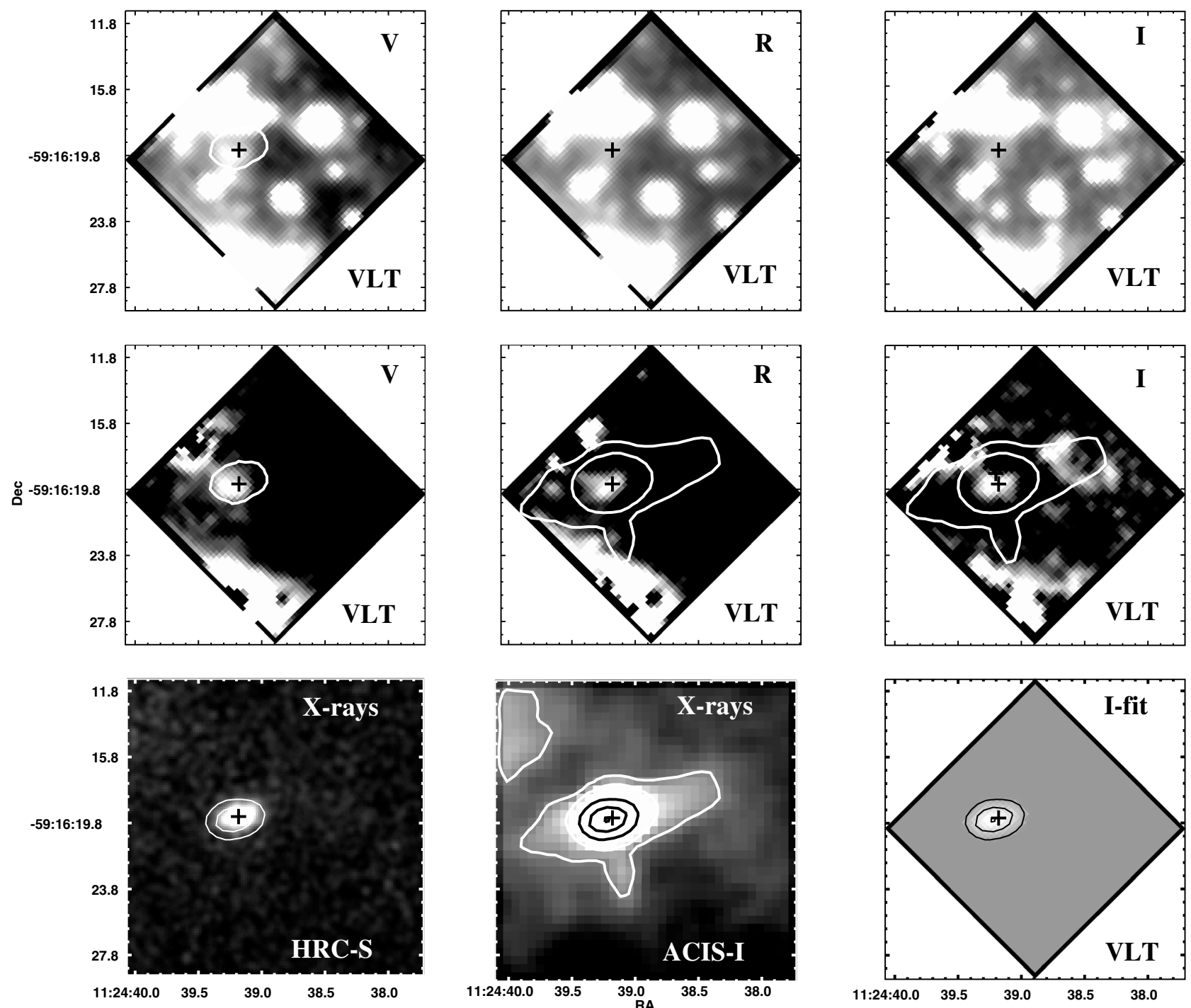

Fig. 2. Central $\sim 16^{\prime \prime} \times 16^{\prime \prime}$ fragment of G292.0+1.8 containing the pulsar J1124-5916 and its PWN. Top panels are VLT/FORS2 $V, R$ and $I$ band optical images, as marked in the panels. Middle panels are the same images, where unsaturated stars have been subtracted. Left and middle bottom panels are the Chandra/HRC-S and ACIS-I X-ray images, respectively. Right bottom panel is the elliptical surface brightness fit of the optical counterpart candidate of the pulsar+PWN system in the $I$ band. Its contours are overlaid on the X-ray images (black on ACIS-I and white on HRC-S). The contours outlining the boundary of the PWN in the HRC-S image and its brightness distribution in the ACIS-I image are overlaid on the $V$ and the star subtracted $R$ and $I$ images, respectively. All images are smoothed with a Gaussian kernel of three pixels. In the optical range this corresponds to a mean seeing value. The cross shows the X-ray position of the pulsar and the sizes of its arms are $1 \sigma$ uncertainties of the position.

dynamical range of the ACIS-I image was changed relative to that shown in Fig. 1 to reveal the structure of the torus-like PWN with the south jet (assuming the torus is seen almost edge-on). Only the central part of the torus with the pulsar is seen on the less deep HRC-S image.

In all three optical bands (top panels of Fig. 2), at the pulsar position marked by a cross, we detect a faint extended object, hereafter named the nebulosity. To study its detailed spatial morphology we show in Fig. 3 a zoomed fragment of the $V$ band image, which is the deepest available one and provides the best spatial resolution (cf., Table 1). The spatial brightness distribution is underlined by contours, demonstrating the east-west extent of the nebulosity and a distinct brightness peak whose position is compatible with the position of the pulsar in X-rays at an $\sim 1 \sigma$ uncertainty level. The north-south wings of the nebulosity partially overlap with the wings of nearby north and south background stars, specifically with the closest one located only $\sim 1$ '. 2 to the south of the pulsar. However, the brightness of its central part cannot be caused by the overlapping wings of these stars. The same conclusion is valid for the $R$ and $I$ images, although they were obtained at about $30 \%$ worse seeing and in these images the nebulosity is more contaminated by the stellar wings. Nevertheless, its central peak is also clearly resolved.

Our limited spatial resolution and the presence of the nearby stars does not allow us to draw definite conclusions on the nebulosity extent in the original images. To better reveal the morphology of the object we produced star-subtracted images. We constructed a model point spread function (PSF) based on a set of about 30 isolated and unsaturated field stars in our images and used the allstar task from the IRAF digiphot tool. The task provides an efficient iterative algorithm for a careful subtraction of groups of stars with overlapping PSFs. The latter is 


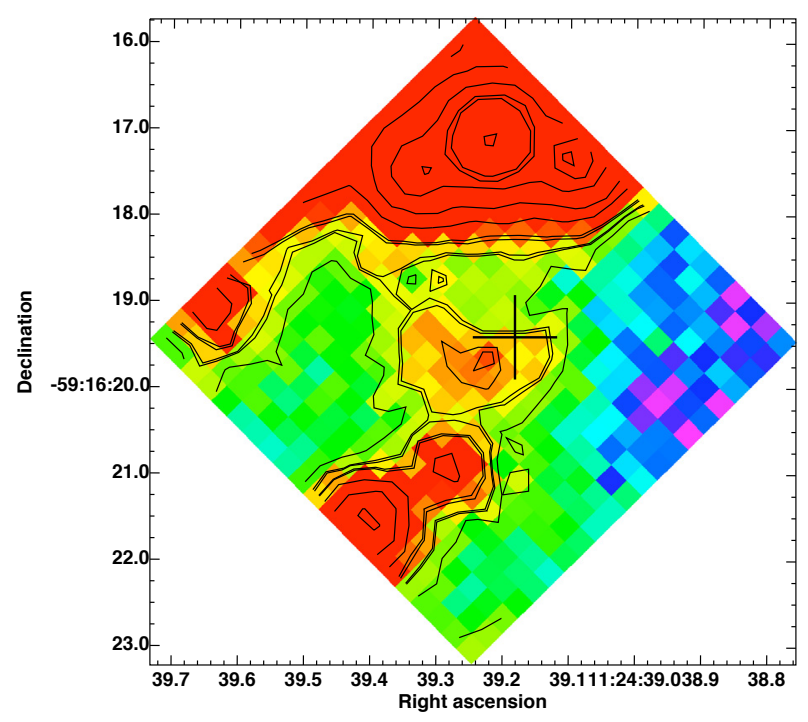

Fig. 3. Zoomed fragment of the $V$ band image shown in Fig. 2. Contours underline the surface brightness distribution. The cross marks the X-ray position of PSR J1124-5916, the sizes of its arms are $2 \sigma$, or $\sim 0$ '. 5 , uncertainties of the position (see text for details).

crucial in our case since most of the stars near the pulsar are in such groups. Since the subtraction process is somewhat imperfect, we repeated it several times, varying the allstar parameters. The results for each of the bands were rather stable and they are shown in the middle panels of Fig. 2. The main contaminating factor, the star $\sim 1^{\prime \prime} .2$ south of the pulsar, as well as other unsaturated stars are perfectly subtracted. That is obviously not possible for saturated stars with corrupted PSFs, whose remains are noticeable. However, they are distant enough from the nebulosity to affect its morphology. On the resulting images, the nebulosity has an ellipsoidal shape compatible with the structure seen in the HRC-S image, revealing only the brightest inner part of the PWN torus with the pulsar. Both the extent of the optical source and the orientation of its major axis are similar to what was found in X-rays. This suggests that the detected source is a likely optical counterpart of the central part of the PSR J11245916+PWN system.

We do not resolve in our optical images any signature of the southern X-ray jet of the PWN. This is not a surprise since the jet is much fainter than the torus and it was clearly resolved in X-rays only after a very deep observation with the ACIS-I (Park et al. 2007).

To compare quantitatively the morphology of the suggested optical counterpart with the X-ray PWN torus structure, we have fitted the spatial intensity distribution in the star-subtracted images with a simple two-dimensional model (using the IRAF isophote task) accounting for the brightness distribution by elliptical isophotes and taking into account the background level. A similar fit has been made by Hughes et al. (2003) to the pulsar/PWN source on the HRC-S image. The results of the fit for the $I$ band are presented in the right bottom panel of Fig. 2 and Table 3 . The fit shows that the brightest inner part of the nebula, within 1 ". -1 ".5 of the center, has almost a circular shape and emits $\approx 20-40 \%$ of the total flux. If our identification is correct, this can be considered as an upper limit of the pulsar contribution to the total pulsar+PWN flux. The coordinates of the center defined from the fit are $\alpha_{2000}=11: 24: 39.216$, and $\delta_{2000}=-59: 16: 19.60$. The measured pulsar centroid positions
Table 3. Parameters of the elliptical fit to the surface brightness of the PSR J1124-5916+PWN optical counterpart ordered by the major ellipse axis length (cf., right bottom panel of Fig. 2).

\begin{tabular}{llllll}
\hline \hline $\begin{array}{l}\text { Center } \\
\text { RA }\end{array}$ & $\begin{array}{l}\text { Center }^{a} \\
\text { Dec }\end{array}$ & $\begin{array}{l}\text { Major } \\
\text { axis } \\
\text { length }\end{array}$ & $\begin{array}{l}\text { Ellip- } \\
\text { ticity }^{b}\end{array}$ & $\begin{array}{l}\text { PA }^{c} \\
\text { W to N }\end{array}$ & $\begin{array}{l}\text { I-band } \\
\text { flux }^{d}\end{array}$ \\
$(11: 24:$ & $(-59: 16:$ & & & & \\
$39 . *)$ & $\left.19 *^{*}\right)$ & (arcsec) & & (degrees) & $(\%)$ \\
\hline 216 & 60 & 1.26 & 0.20 & -1.3 & $24.9(7.6)$ \\
230 & 59 & 1.50 & 0.25 & -0.2 & $46.4(8.1)$ \\
240 & 61 & 1.76 & 0.37 & 13.2 & $51.8(8.2)$ \\
235 & 59 & 2.00 & 0.34 & 11.5 & $66.2(8.6)$ \\
211 & 57 & 2.50 & 0.50 & 12.8 & $76.5(8.8)$ \\
212 & 57 & 2.76 & 0.48 & 10.3 & $87.3(9.3)$ \\
212 & 57 & 3.01 & 0.48 & 10.3 & $96.8(9.8)$ \\
212 & 57 & 3.21 & 0.48 & 10.3 & $100(7.1)$ \\
\hline
\end{tabular}

${ }^{a}$ Coordinates of centers of the ellipses $(\mathrm{J} 2000) ;{ }^{b}$ defined as $1-l_{\min } / l_{\max }$, where $l_{\min }$ and $l_{\max }$ are the minor and major ellipse axis lengths, respectively; ${ }^{c}$ positional angle of the major axis; ${ }^{d}$ flux from the elliptical aperture normalized to the flux from the largest aperture in this set. Numbers in brackets are $1 \sigma$ uncertainties.

in X-rays are $\alpha_{2000}=11: 24: 39.183, \delta_{2000}=-59: 16: 19.41$ and $\alpha_{2000}=11: 24: 39.180, \delta_{2000}=-59: 16: 19.49$, as obtained from the HRC-S ${ }^{7}$ and ACIS-I images, respectively. Both are in good agreement with each other and with the center position of the optical source taking into account the $\sim 0$ ' $^{\prime} 15$ and $\sim 0 . ' 2$ astrometric accuracy in the optical and X-rays (Sect. 2.2). The ellipticity of nebulosity increases up to $\sim 0.5$ for the outward optical nebula regions and the major axis is slightly tilted from the RA axis by about $10^{\circ}-13^{\circ}$, which is also in good agreement with the about $13^{\circ}$ tilt seen in X-rays. In addition, the optical nebula is elongated almost symmetrically around its center with a total length of $\sim 33^{\prime \prime} 2$, which agrees with the extent of the brightest part of the PWN seen in X-rays. Assuming that we see a tilted circular torus, we estimate the torus axis angle to the line of sight in the range of $50^{\circ}-60^{\circ}$.

Extending the analysis of the nebular structure in the optical and X-rays we also considered $1 \mathrm{D}$-spatial profiles of the images along the two slices directed along the nebula symmetry axes. The slice positions are shown in the left panel of Fig. 4. Their widths are 1 ", both centered on the the X-ray pulsar position. A "horizontal" slice is 6" long and placed symmetrically over the pulsar position to include most of the emission along the major axis of the nebula. Its PA to the RA axis is $\approx 13^{\circ}$. The "vertical" slice is orthogonal to the first one, it is $8^{\prime \prime}$ long and extended towards the south-south-west to also include the X-ray jet. The respective 1D-profiles performed on the star-substracted images are shown in the middle and right panels of Fig. 4. These show the coincidence in the positions of the main peaks of the nebula and their extents in the optical and X-rays. The ACIS-I peak is broader than that of the HRC-S because of the lower spatial resolution of ACIS. A wing asymmetry of the $V$ and $R$ peaks in the middle panel and the flux enhancement in the southern part of the all optical profiles in the right panel are not related to the nebula. These are subtraction remains of nearby background stars east and south of the PWN, respectively. The southern jet, barely resolved in the ACIS-I profile in the right top panel, cannot be reliably resolved in the optical profiles in part because of

\footnotetext{
7 Our HRC-S coordinates, when rounded by the last two digit numbers, are compatible with the published ones (Hughes et al. 2003).
} 

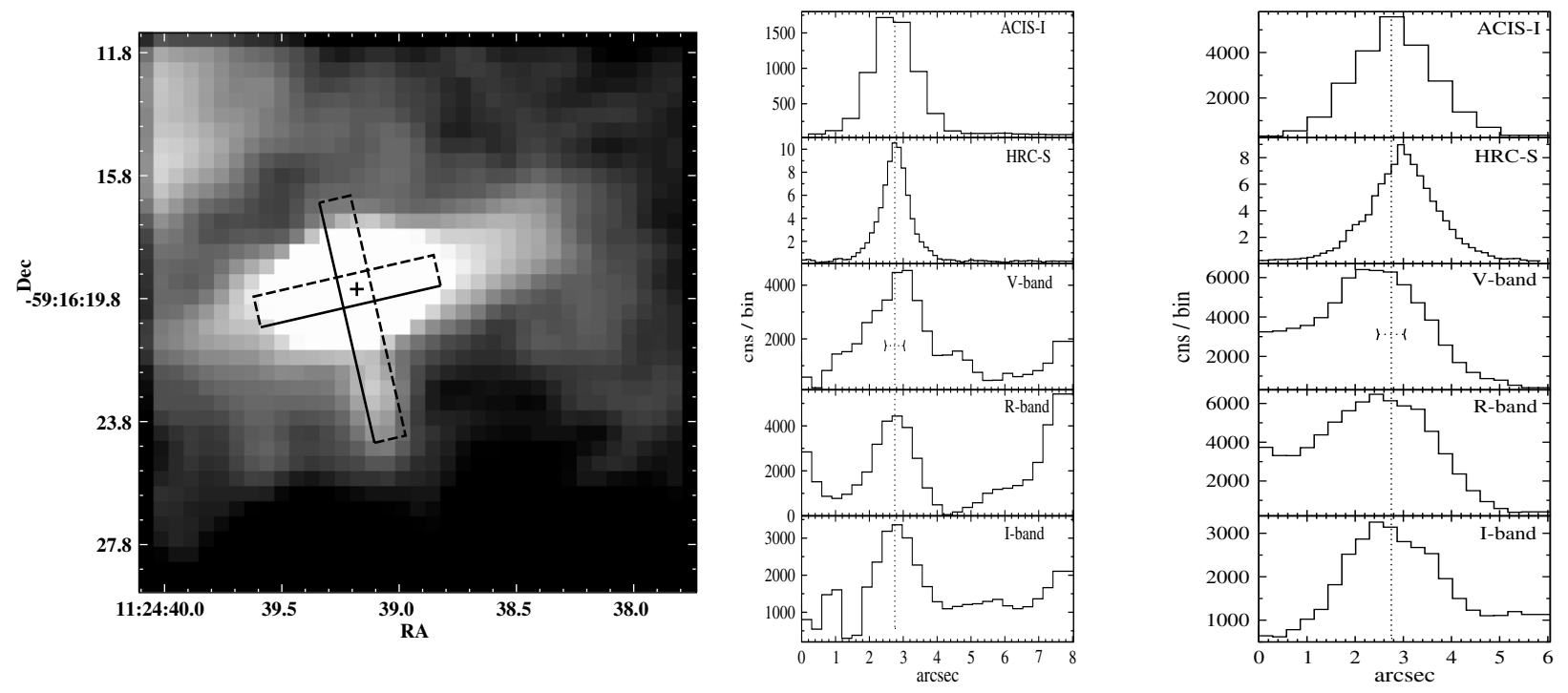

Fig. 4. Left: the ACIS-I image with positions of two slices (bold lines) used for comparison of 1D X-ray source profiles of the J1124-5916 pulsar+PWN system with its optical counterpart candidate profiles; cross marks the position of the pulsar. Middle and right: the source profiles extracted from the ACIS-I, HRC-S, $V, R$, and $I$ images, as marked in the panels, along the "horizontal" and "vertical" slices, respectively, shown on the left. The coordinate origins of the horizontal axis in the middle and right panels coincide with the eastern and northern edges of the respective slices. Vertical dotted lines with horizontal error-bars mark the X-ray position of the pulsar and its uncertainties.

the asymmetry of the contaminating effect of the above remains.

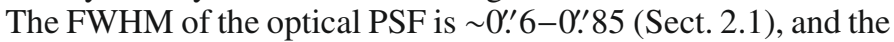
brightest part of the nebula, which extends to several arcseconds, is clearly resolved. Although the position of the main optical peak coincides with the pulsar position, we do not resolve any point-like source at this position. To resolve the point-like pulsar from the nebula would require deeper imaging at higher spatial resolution, and/or time resolved observations.

We can conclude that our spatial analysis strongly favors the suggested optical identification of the G292.0+1.8 pulsar/PWN system. The main uncertainties are related to a possible imperfect subtraction of the closest stars contaminating the emission of the outer parts of the PWN optical counterpart candidate. This may affect the derived extension of the optical source along the minor axis of the X-ray PWN torus and the parameters of the elliptical surface brightness fit. However, the similarity of the counterpart candidate morphology in the star-subtracted images in the different optical bands, where the star appearances are considerably different, supports the correctness of the performed subtraction and the PWN identification. Higher spatial resolution imaging is needed to confirm this.

\subsection{Photometry}

The optical photometry of the suggested counterpart was performed on the star-subtracted images. We used the elliptical apertures from the surface brightness fit described in Sect. 3.1. Their parameters are presented in Table 3. The background levels were estimated from a circular annulus with $\sim 7$ pixel inner radius and a width of $\sim 5$ pixels centered on the nebula center. The relative photometric errors were minimal $(S / N \approx 13)$ for apertures formally encapsulating $\gtrsim 90 \%$ of the total nebula flux. The measured integral magnitudes of the presumed pulsar+torus nebula are summarized in Table 4, where the errors include the calibration zero-point uncertainties, statistical measurement errors, and account for possible star subtraction uncertainties based on the magnitude dispersion after the reiterations
Table 4. Observed magnitudes and fluxes for the presumed optical pulsar/PWN counterpart in G292.0+1.8, as well as de-reddened fluxes for different $A_{V}$ values.

\begin{tabular}{llllll}
\hline \hline Band & $\begin{array}{l}\text { Mag. } \\
\text { obs }^{a}\end{array}$ & $\begin{array}{l}\text { log } F \\
\operatorname{obs}^{a}\end{array}$ & & \multicolumn{3}{c}{$\begin{array}{l}\log F \\
\operatorname{dered}^{a, b}\end{array}$} \\
\cline { 4 - 6 } & $(\mathrm{mag})$ & $(\mu \mathrm{J})$ & $A_{V}=1.86$ & $\begin{array}{l}2.06(4) \\
(\mu \mathrm{J})\end{array}$ & $1.86-2.1$ \\
\hline$V$ & $24.29(13)$ & $-0.16(5)$ & $0.58(5)$ & $0.66(7)$ & $0.66\left(_{-13}^{+7}\right)$ \\
$R$ & $24.12(13)$ & $-0.17(5)$ & $0.44(5)$ & $0.51(7)$ & $0.51\left(_{-22}^{+7}\right)$ \\
$I$ & $23.12(13)$ & $0.13(5)$ & $0.61(5)$ & $0.66(6)$ & $0.66\left(_{-10}^{+6}\right)$ \\
\hline
\end{tabular}

${ }^{a}$ Numbers in brackets are $1 \sigma$ uncertainties referring to the last significant digits quoted; ${ }^{b}$ uncertainties include $A_{V}$ uncertainties.

of the subtraction process. We also tried circular and polygonal apertures to better encapsulate the whole flux, but got practically the same results. The stellar magnitudes were transformed into fluxes using zero-points provided by Fukugita et al. (1995).

The observed color indices of the nebulosity are $V-R=$ 0.2 (2) and $R-I=1.0(2)$. We also measured the indices of a random set of faint field stars located, as is our nebulosity, outside the known emission line regions of the SN remnant (Winkler \& Long 2006; Park et al. 2007). We obtained indices in the range of $0.6 \leq V-R \approx R-I \leq 1.1$. This corresponds to the stellar "reddened" color indices and it is significantly different from the nebulosity colors. Based on that, the detected nebulosity is unlikely to be a result of a superposition of a few overlapping, unresolved faint background stars.

\subsection{Multi-wavelength spectrum of the pulsar+PWN source}

Using the optical fluxes for the pulsar/PWN counterpart candidate together with the X-ray data we can construct a tentative multi-wavelength spectrum of the central part of the source. 
To compare our optical fluxes with the X-ray spectrum from the same physical region of the pulsar+PWN system we applied the 3'.2 major axis length elliptic aperture from Table 3 that encircles $\sim 100 \%$ of the optical flux. Using the ACIS-I Obs Ids 6677 and 6679 with the longest individual exposures $(\sim 161$ and $\sim 156 \mathrm{ks})$ of the G292.0+1.8 center and the CIAO acisspec tool, within this elliptical aperture we obtained 7659 and 7393 source counts, respectively. A circular region of $\sim 10^{\prime \prime}$ diameter located $\sim 16^{\prime \prime}$ south of the pulsar was used to estimate the background contribution, which was $\$ 1 \%$ within the source aperture. Spectral data of both OBs were grouped to provide a minimum 25 counts per spectral bin and fitted simultaneously by an absorbed power-law model using standard XSPEC tools. As a result, we obtained a statistically acceptable fit with a spectral in$\operatorname{dex} \Gamma=1.851 \pm 0.027$, absorbing column density $N_{\mathrm{H}}=(0.369 \pm$ $0.014) \times 10^{22} \mathrm{~cm}^{-2}$ and normalization constant $\mathrm{C}=(1.38 \pm$ $0.08) \times 10^{-4}$ photons $\mathrm{cm}^{-2} \mathrm{~s}^{-1} \mathrm{keV}^{-1}$. The fit had $\chi^{2}=0.962$ per degree of freedom and the unabsorbed integral flux was $5.626 \times$ $10^{-13} \mathrm{erg} \mathrm{cm}^{-2} \mathrm{~s}^{-1}$ in the $0.3-10 \mathrm{keV}$ range.

The fit residuals do not show any signs of emission lines, as found by Hughes et al. (2001) with a few arcseconds larger aperture. This means that the spectrum is unlikely to be contaminated by the emission form the supernova ejecta. The derived spectral index is also compatible with typically observed indices for Crab-like PWNe. We checked our result using the same aperture and counts per bin setup on previous $\sim 44 \mathrm{ks}$ observations of G292.0+1.8 with the ACIS-S ${ }^{8}$. Within the larger uncertainties resulting from lower count statistics at this short exposure we obtained the same fit parameters. This confirmed that for the selected pulsar+PWN region both the ACIS-I and the ACIS-S provide the same results. Finally, we adopted a circular aperture of 2.5 pixel radius $\left(1^{\prime \prime} .23\right)$ centered on the pulsar position, as used by Hughes et al. (2001), to estimate the spectrum of the pulsar on the ACIS-S data. Within uncertainties, both the ACIS-S and the ACIS-I gave a spectral index and the column density consistent with those obtained for the elliptical aperture. For instance, for the ACIS-I we have $\Gamma=1.851 \pm 0.027$ and $N_{\mathrm{H}}=(0.367 \pm$ $0.014) \times 10^{22} \mathrm{~cm}^{-2}$. This is in contrast to smaller values, $\Gamma=$ $1.72 \pm 0.05$ and $N_{\mathrm{H}}=(0.317 \pm 0.015) \times 10^{22} \mathrm{~cm}^{-2}$, published by Hughes et al. (2001). We have been able to reproduce the published values only for the case of the ACIS-S data when the spectral range at the fit was constrained by $0.3-7 \mathrm{keV}$. A similar constraint for the ACIS-I data with much higher count statistics does not lead to any significant spectral hardening and/or $N_{\mathrm{H}}$ decrease. This shows that the PWN contribution to the total pulsar+PWN flux is still rather high even for such a small aperture and the previous estimates of the pulsar spectrum and $N_{\mathrm{H}}$ based on the short ACIS-S exposure may be unreliable.

The next step in the multi-wavelength spectrum analysis is the correction of the optical fluxes for interstellar reddening. Compiling various hydrogen column density measurements in the radio and $\mathrm{X}$-rays and comparing the observed optical spectral-line intensity ratios for G292.0+1.8 filaments with theoretical models of the post shock line emission from the material ejected from the SN progenitor, Winkler \& Long (2006) adopted the interstellar color excess of $E(B-V)=0.6 \mathrm{mag}$. On the other hand, using the $N_{\mathrm{H}}$ value derived from our X-ray spectral fit and an empirical relation between the effective $N_{\mathrm{H}}$ of the X-ray absorbing gas and the dust extinction $N_{\mathrm{H}} / E(B-V)=$ $(5.55 \pm 0.093) \times 10^{21} \mathrm{~cm}^{-2} \mathrm{mag}^{-1}$ (Predehl \& Schmitt 1995), we obtain a larger value of $E(B-V)=0.665 \pm 0.014 \mathrm{mag}$, which corresponds to the $V$-band reddening correction

\footnotetext{
${ }^{8}$ OBs ID 126, exposure 44 ks, PI Garmaire.
}

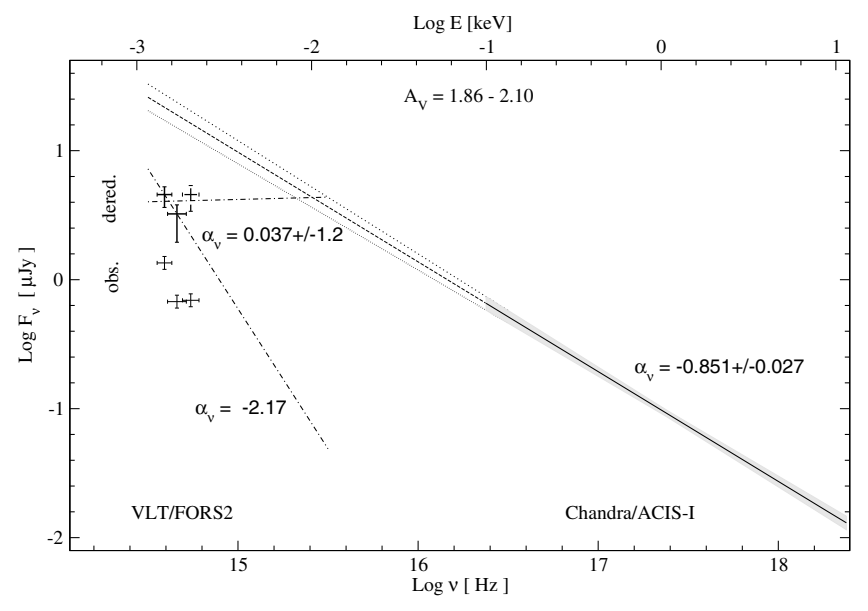

Fig. 5. Tentative unabsorbed multi-wavelength spectrum for the inner part of the torus region of the G292.0+1.8 pulsar/PWN system compiled from the data obtained with the VLT and Chandra, as indicated in the plot. Thin cross-bars, marked by "obs.", show the observed VRI optical fluxes to demonstrate the interstellar extinction effect. Error-bars for the de-reddened fluxes, marked as "dered", include the $A_{V}$ uncertainty range shown at the top of the plot. The $V$ band flux (dashed cross-bars) is likely contaminated by strong OIII emission from the SNR and may be considered as a flux upper limit for the pulsar/PWN system. The grey polygon demonstrates the spectral uncertainty in X-rays. It is extrapolated towards the optical together with the best X-ray power-law spectral fit (solid and dashed lines). As seen, the extrapolated spectrum strongly overshoots the optical fluxes, suggesting at least one spectral break between the optical and X-rays. A crude power-law fit to the optical data are shown by dot-dashed lines for two cases: when all three optical bands are included in the fit (upper line); and when the $V$ band flux is excluded from the fit (lower line with a higher slope). $\alpha_{v}$ are spectral indices of the power-law fits defined as $F_{v} \propto v^{\alpha_{v}}$.

$A_{V}=2.062 \pm 0.043 \mathrm{mag}$, adopting a standard ratio $A_{V} / E(B-$ $V)=3.1$. Within $5 \sigma$ this is consistent with the above $E(B-$ $V)$ value $\left(A_{V}=1.86 \mathrm{mag}\right)$ and significantly smaller than the extinction of 0.86 mag caused by whole Galaxy in this direction (Schlegel et al. 1998). De-reddened optical fluxes for the considered $A_{V}$ values and their combination are presented in Table 4. For de-reddening we used a standard optical extinction curve (Cardelli et al. 1989).

A tentative unabsorbed multi-wavelength spectrum of the central part of the pulsar/PWN system is compiled in Fig. 5. For this plot, we selected an $A_{V}$ range of 1.86-2.10 mag, which covers the whole range of reasonable extinction values discussed above. The error-bars for the dereddened optical fluxes include this range, while the centers of the cross-bars correspond to dereddenings with $A_{V} \approx 2.06$ derived from $N_{\mathrm{H}}$ given by our X-ray spectral fit (cf. Table 4). We note that the resulting dereddened flux errors are strongly dominated by the $A_{V}$ uncertainties, and any uncertainties introduced by the star subtraction process are insignificant at this level. As seen, the extrapolation of the X-ray power-law spectral fit to the optical range strongly overshoots the optical fluxes. The coincidence can be achieved only at unreasonably high extinction $A_{V} \gtrsim 3.6 \mathrm{mag}$, which is significantly higher than the entire Galactic extinction in this direction, $A_{V} \approx$ 2.7 (Schlegel et al. 1998). This suggests at least one spectral break between the optical and X-rays. It is also evident for the pulsar spectrum if we are to combine the X-ray data and the optical flux upper limit obtained early by Hughes et al. (2004).

The actual number of the breaks depends on the real spectral energy distribution (SED) in the optical range. As seen, both the observed and dereddened SEDs are apparently non-monotonic. 
One plausible reason is a contamination of the measured continuum emission form the pulsar/PWN in the $V$ band by a strong background [OIII] emission from the oxygen-rich SN remnant knots and filaments (Winkler \& Long 2006). Some emission clumps visible in this band south of the suggested counterpart also may be associated with faint [OIII] knots. Their presence near the pulsar position cannot be excluded considering the available low spatial resolution narrow-band [OIII] images published by Winkler \& Long (2006). Other emission lines (e.g., $\mathrm{H}_{\alpha}$, [OI], [OII] and [SII]) detected from the $\mathrm{SN}$ remnant in the red part of the spectrum enter the $R$ and $I$ passbands. However, they are much fainter than that of [OIII] and cannot significantly contaminate the respective parts of the continuum emission expected to be produced by the pulsar and its PWN. A similar situation has been observed in another young pulsar, PSR B0540-69, which is also associated with an oxygen-rich SNR B0540-69.3 in the LMC (Serafimovich et al. 2004). In this case, the flux in the $V$ band can be considered only as an upper limit for the pulsar/PWN emission. To underline this we represent the respective dereddened flux by a dashed cross-bar in Fig. 5. If we exclude the $V$ band, then the optical part of the spectrum becomes much steeper $\left(\alpha_{v} \approx-2.2\right)$ than in X-rays $\left(\alpha_{v} \approx-0.85\right)$, which suggests a double knee spectral break between these two ranges, as is observed for the PSR B0540-69/PWN system. Alternatively, if we take into account the $V$ band as well, the resulting optical spectrum can be flat or less steep, which leads to a single spectral break, as is observed for the Crab PWN (e.g., Serafimovich et al. 2004; Sollerman 2003).

So far only the Crab and PSR B0540-69 PWNe have been firmly detected in the optical range. Within the uncertainties the shape of the tentative multi-wavelength spectrum of the PSR J1124-5916/PWN system is either similar to one of the above systems or intermediate between them. This is additional evidence of the correct optical identification.

\section{Discussion}

The deepest up to date broadband optical observations of the PSR J1124-5916 field with the VLT reveal a faint nebulosity at the pulsar position. The sub-arcsecond agreement of its brightness peak position with the pulsar coordinates, the likely similar optical source morphology to that of the brightest inner part of the G292.0+1.8 X-ray torus-like PWN, and the consistence of its tentative multi-wavelength spectrum with the spectra of other PWNe allow us to consider the detected source as a very likely optical counterpart of the G292.0+1.8 pulsar+PWN system. The nebulosity appearance in the VRI bands excludes the possibility that it is due to either a group of a few close unresolved faint field stars or overlapping wings of the nearby detected stars.

Alternatively it could be a SNR filament, or a faint distant background galaxy coinciding by chance with the pulsar position. However, no strong optical and X-ray emission lines typical of SN remnant filaments were found within the central part of the PWN torus region (Winkler \& Long 2006; Park et al. 2007). This makes the filament interpretation rather unlikely. To confirm this, we have analyzed the torus regions extended up to $\sim 14$ ". $8 \times$ 8.' 6 elliptical boundary using X-ray ACIS-I data. A combination of the absorbed power-law and vmekal spectral models and elliptical annular apertures centered on the pulsar was used. The contribution of the vmekal becomes statistically significant only outside the $\approx 9$ '. $6 \times 4$ ". 8 elliptical boundary, improving the spectral fit by the appearance of the $\mathrm{O}, \mathrm{Mg}$, and $\mathrm{Si}$ emission lines from the soft thermal radiation of a hot $\mathrm{SN}$ remnant gas. This is roughly consistent with the $\sim 5^{\prime \prime} \times 3^{\prime \prime}$ boundary, obtained by
Hughes et al. (2001) based on previous ACIS-S data. Therefore, the strong line emission, typical of filaments, is at least outside the limits of the optical source and of the elliptical aperture used to analyze the X-ray non-thermal emission from the internal torus region. On the other hand, further observations in the near-infrared and near-UV are necessary to better constrain the SED of the nebulosity and to distinguish it from a possible background galaxy. The power law SED in a wide spectral range would be a strong confirmation of the PWN origin. This would also enable us to better constrain the extinction value towards the source and to check whether the apparent flux excess in the $V$ band is indeed due to the contamination from a faint and not yet resolved background [OIII] emission of the SN remnant.

Assuming that the pulsar contribution to the measured optical fluxes $\$ 20 \%$, based on the spatial profiles of the nebulosity and on its surface brightness fits, the predicted optical magnitudes of the point-like pulsar counterpart would be $V \gtrsim 26^{\mathrm{m}} 2$, $R \gtrsim 26$. $0, I \gtrsim 25$. 0 . These are quite reachable with current telescope capacities. However, higher spatial resolution is necessary to resolve such faint point-like object over the extended source background and also is important to minimize the contamination of the nebulous emission from the closest field stars. Deeper optical observations may help to reveal outer fainter regions of the nebulosity at larger spatial scale comparable to the whole X-ray PWN extent.

There are no reports of radio detections from the central torus region of J1124-5916 PWN. On larger spatial scales, the G292.0+1.8 radio plerion shows a core whose brightness peak is located about 30" north of the pulsar (Gaensler et al. 2002), while the pulsar is surrounded by fainter emission. It is interesting that the internal region of this radio core is extended in the direction (see Fig. 3 from Gaensler et al. 2002) roughly parallel to the major axis of the elliptical PWN torus region. At the same time, the outer contours of the radio core are extended in the orthogonal direction, i.e., south-south-west, which may be associated with the direction of the X-ray jet propagating along the torus symmetry axis. This multi-wavelength structure of the J1124-5916 PWN appears to be similar to what is seen for the Crab plerion that has a larger extent, namely along the PWN torus symmetry axis. High resolution radio observations of G292.0+1.8 would be useful to understand if the suggested similarity really exists. Such observations would also allow us to constrain the radio flux from the compact PWN torus and its spectral slope in the radio range.

Assuming that we have detected the optical counterpart of the torus-shaped PWN of G292.0+1.8, we compiled in Table 5 the properties of this and other young PWNe detected in the optical and X-rays ${ }^{9}$. We also include the older Vela, whose PWN has not yet been identified in the optical range (Mignani et al. 2003; Shibanov et al. 2003). For G292.0+1.8 we adopt the distance of $6 \mathrm{kpc}$ (Gaensler et al. 2002), and the uncertainty of its tentative PWN optical luminosity is mainly due to the uncertainty in the interstellar extinction. We also updated the total X-ray luminosity of this PWN using the new ACIS-I data and an elliptical aperture encapsulating the whole PWN torus flux. As a result, the updated flux is by an order of magnitude lower than was estimated previously by Hughes et al. (2001) using the ACIS-S data and assuming that the G292.0+1.8 PWN spectral index is the same as for the Crab PWN (see also Kargaltsev \& Pavlov 2008). In the $2-10 \mathrm{keV}$ image extracted from the ACIS-I data, which is not contaminated by radiation from strong SNR lines, one can resolve a faint diffuse non-thermal emission around the

\footnotetext{
9 Abbreviations: 0540 is B0540-69; J1124 is J1124-5916.
} 
Table 5. Comparison of the optical and X-ray spectral indices $\left(\alpha_{v}^{\mathrm{O}}, \alpha_{v}^{\mathrm{X}}\right)$ luminosities $\left(L^{\mathrm{O}}, L^{\mathrm{X}}\right)$, and efficiencies $\left(\eta^{\mathrm{O}}, \eta^{\mathrm{X}}\right)$ of the three young PWNe detected in the optical/IR and X-rays. Information on the Vela PWN, not yet detected in the optical, as well as on the pulsar characteristic ages $(\tau)$, spindown pulsar luminosities $(\dot{E})$, PWN sizes, and the ratios of the pulsar to PWN luminosity in the optical and X-rays are also included.

\begin{tabular}{lccccccccccc}
\hline \hline $\mathrm{PWN}$ & $\begin{array}{c}\tau \\
\mathrm{kyr}\end{array}$ & $\begin{array}{c}\dot{E} \\
10^{37} \mathrm{erg} \mathrm{s}^{-1}\end{array}$ & $\begin{array}{c}\mathrm{size} \\
\mathrm{pc}\end{array}$ & $-\alpha_{v}^{\mathrm{O}}$ & $\begin{array}{c}L^{\mathrm{O}, \mathrm{a}} \\
10^{33} \mathrm{erg} \mathrm{s}^{-1}\end{array}$ & $\begin{array}{c}\eta^{\mathrm{O}} \\
10^{-5}\end{array}$ & $-\alpha_{v}^{\mathrm{X}}$ & $\begin{array}{c}L^{\mathrm{X}, \mathrm{b}} \\
10^{36} \mathrm{erg} \mathrm{s}^{-1}\end{array}$ & $\begin{array}{c}\eta^{\mathrm{X}} \\
10^{-3}\end{array}$ & $\begin{array}{c}L_{\mathrm{psr}}^{\mathrm{O}} / L_{\mathrm{pwn}}^{\mathrm{O}} \\
L_{\mathrm{psr}}^{\mathrm{X}} / L_{\mathrm{pwn}}^{\mathrm{X}}\end{array}$ \\
\hline $\mathrm{Crab}^{c}$ & 1.24 & 46 & 1.5 & 0.92 & 4240 & 920 & 1.14 & 21.8 & 47.5 & 0.0017 & 0.046 \\
$0540^{c}$ & 1.66 & 50.2 & $0.6-0.9$ & 1.5 & 366 & 245 & 1.04 & 12 & 79.7 & 0.03 \\
$\mathrm{~J}_{1124^{d}}$ & 2.9 & 1.2 & $0.2-0.4$ & $0.0-2.2$ & $0.02-0.09$ & $0.17-0.76$ & 0.85 & $4.2 \times 10^{-3, f}$ & 0.34 & $\vdots 0.2$ & 0.26 \\
$3 \mathrm{C} 58^{e}$ & 5.38 & 2.6 & $0.08-0.19$ & $0.7-1.2$ & $0.08-0.21$ & $0.3-0.75$ & 0.88 & $5 \times 10^{-3}$ & 0.19 & $\lesssim 0.1$ & 0.23 \\
$\mathrm{Vela}^{c}$ & 11 & 0.069 & 0.14 & - & - & - & 0.5 & $6.8 \times 10^{-4}$ & $9.8 \times 10^{-2}$ & - & 0.34 \\
\hline
\end{tabular}

${ }^{a}$ For the optical range $1.57-3.68 \mathrm{eV} ;{ }^{b}$ for the X-ray range $0.6-10 \mathrm{keV} ;{ }^{c}$ data are taken from Serafimovich et al. (2004); ${ }^{d}$ this paper. The distance is assumed to be $6.0 \mathrm{kpc} ;{ }^{e}$ data are taken from Shibanov et al. (2008); ${ }^{f}$ this paper. For the whole torus region.

PWN extending $\sim 30^{\prime \prime}-40^{\prime \prime}$ from the pulsar, partially overlapping with the radio core region. Accounting for this emission in the power law spectral fit with $N_{\mathrm{H}}$ fixed at the value derived for the central torus region, we obtained an upper limit for the whole non-thermal X-ray luminosity of the plerion $L_{\text {plerion }} \approx 3 \times$ $10^{34} \mathrm{erg} \mathrm{s}^{-1}$ (in $0.5-8 \mathrm{keV}$ range). Compared with the whole torus luminosity, we see that this faint but largely extended diffusive emission can contribute up to $85 \%$ of the total plerion luminosity. However, this value is a factor of two lower than early PWN luminosity estimates. The PSR J1124-5916 X-ray luminosity is taken from Hughes et al. (2003), where the detection of the X-ray emission with the pulsar period was reported. Our estimates show that it can be uncertain by a factor of two. The respective value published later by Kargaltsev \& Pavlov (2008) actually coincides with the luminosity of the whole torus+pulsar system and, hence, is strongly overestimated. In Table 5 the pulsars are listed in characteristic age order. As seen, J1124-5916 fits well into the correlation of the X-ray and optical "strength" of PWNs with the spin-down luminosity. This also supports its correct optical identification. The X-ray and tentative optical parameters of J1124-5916 are very similar to the parameters of the $3 \mathrm{C} 58$ pulsar+PWN system, recently identified in the optical and mid-infrared (Shibanov et al. 2008; Slane et al. 2008; Shearer \& Neustroev 2008). The coincidence of the properties of these two PWNe is not surprising, since their pulsars have similar key parameters like the spindown luminosity and age. By chance, both torus-like PWNe are seen almost edge-on and display one-sided jets along the torus symmetry axis. Thus, these two PWNe look like twins, although the properties of the associated SNRs are very different. G292.0+1.8 is oxygen rich, while 3C 58 is not, suggesting different masses of the SN progenitor stars.

Finally, we check whether the lower limit obtained here for the optical luminosity of PSR J1124-5916 and that of PSR J0205+6449 from Shibanov et al. (2008) are compatible with the empirical relation between the non-thermal optical and $\mathrm{X}$-ray efficiencies of the pulsars detected in both spectral domains (Zharikov et al. 2004, 2006, 2008). This is shown in Fig. 6. As seen, the optical and X-ray data for the two pulsars do not contradict the relation. Both PSR J1124-5916 and PSR J0205+6449 naturally occupy an intermediate position in the plot between the younger Crab and B0540-69 pulsars and the older Vela, all of which power the torus-like PWNe. This ensures that the optical upper limits for both pulsars are realistic and close to the expected flux values based on their X-ray and spindown luminosities. This encourages further optical searching of both pulsars.

In summary, our observations of the PSR J1124-5916 field likely increase the number of PWNe identified in the optical range from three to four. Additional evidence of the suggested

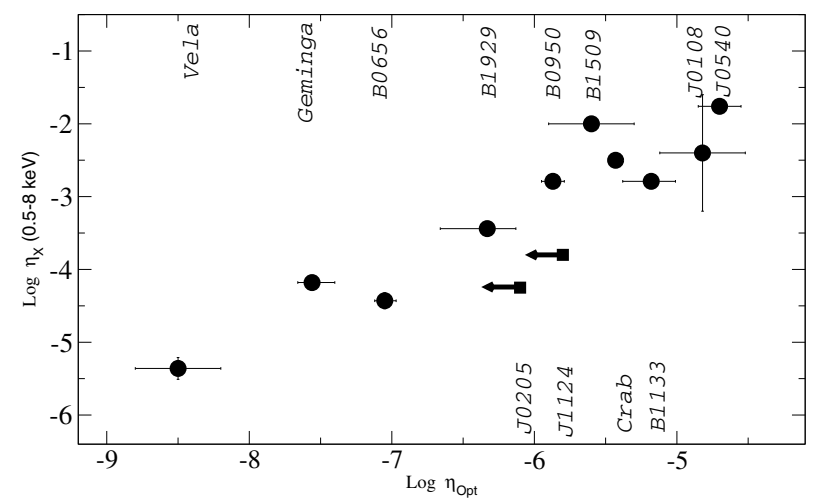

Fig. 6. Relationship between the optical and X-ray efficiencies from Zharikov et al. (2006) updated with data of PSR B1509-58 (Wagner \& Seifert 2000), PSR B1133+14 (Zharikov et al. 2008), PSR J0205+6449 (Shibanov et al. 2008), and PSR J0108-1431(Mignani et al. 2008). X-ray efficiencies $(0.5-8 \mathrm{keV})$ are based on non-thermal pulsar X-ray luminosities from Kargaltsev \& Pavlov (2008) and Pavlov (private communication).

identification should be provided by high spatial resolution optical observations. Together with the data in other spectral domains this should help in constraining models and understanding the emission of these objects.

Acknowledgements. We are grateful to the anonymous referee for useful comments improving the paper. The work was partially supported by CONACYT 48493 and PAPIIT IN101506 projects, RFBR (grants 08-02-00837a, 05-02-22003), NSh-2600.2008.2 and by by the German Deutsche Forschungsgemeinschaft, DFG project number Ts $17 / 2-1$. We used the USNOFS Image and Catalogue Archive operated by the United States Naval Observatory, Flagstaff Station (http://www.nofs.navy.mil/data/fchpix/). The Munich Image Data Analysis System is developed and maintained by the European Southern Observatory. IRAF is distributed by the National Optical Astronomy Observatories, which are operated by the Association of Universities for Research in Astronomy, Inc., under cooperative agreement with the National Science Foundation.

\section{References}

Camilo, F., Manchester, R. N., Gaensler, B. M., et al. 2002, ApJ, 567, L71 Cardelli, J. A., Clayton, G. C., \& Mathis, J. S. 1989, ApJ, 345, 245 Chevalier, R. 2005, ApJ, 619, 839

Fukugita, M., Shimasaku, K., \& Ichikawa, T. 1995, PASP, 107, 945

Gaensler, B. M., \& Wallace, B. J. 2003, ApJ, 594, 326

Gaensler, B. M., Arons, J., Kaspi, V. M., Pivovaroff, M. J., \& Kawai, N. 2002, ApJ, 569, 878

Hughes, J. P., Slane, P. O., Burrows, D. N., et al. 2001, ApJ, 559, L153

Hughes, J. P., Slane, P. O., Park, S., et al. 2003, ApJ, 591, L139

Hughes, J. P., Friedman, R. B., Slane, P. O., \& Park, S. 2004, Young Neutron Stars and Their Environments, held as part of the IAU General Assembly, 
14-17 July, 2003 in Sydney, Australia, ed. F. Camilo, \& B. M. Gaensler (San Francisco, CA: ASP), IAU Symp., 218, 199

Hwang, U., Holt, S., \& Petre, R. 2000, ApJ, 537, L119

Kargaltsev, O., \& Pavlov, G. G. 2008 [arXiv:0801.2602]

Landolt, A. 1992, AJ, 104, 340

Mignani, R. P. 2005, Proc. of The Electromagnetic Spectrum of Neutron Stars, ed. A. Baykal et al. (Springer), 210, 133

Mignani, R. P., De Luca, A., Kargaltsev, O., et al. 2003, ApJ, 594, 419

Mignani, R. P., Pavlov, G. G., \& Kargaltsev, O. 2008, A\&A, 488, 1027

Park, S., Hughes, J. P., Slane, P. O., et al. 2007, ApJ, 670, L121

Predehl, P., \& Schmitt, J. H. M. M. 1995, A\&A, 293, 889

Safi-Harb, S., \& Gonzalez, M. E. 2002, in X-rays at Sharp Focus, ed. E. Schlegel, \& S. D. Vrtilek, Chandra Science Symp. ASP Conf. Ser., 262

Schlegel, D. J., Finkbeiner, D. P., \& Davis, M. 1998, ApJ, 500, 525

Serafimovich, N., Shibanov, Yu. A., Lundqvist, P., \& Sollerman, J. 2004, A\&A, 425,1041

Shearer, A., \& Neustroev, V. V. 2008, MNRAS, 390, 235
Shibanov, Yu. A., Koprsevich, A. B., Sollerman, J., \& Lundqvist, P. 2003, A\&A, 406, 645

Shibanov, Yu. A., Lundqvist, N., Lundqvist, P., Sollerman, J., \& Zyuzin, D. 2008, A\&A, 486, 273

Slane, P., Helfand, D. J., Reynolds, S. P., et al. 2008, ApJ, 676, L33

Sollerman, J. 2003, A\&A, 406, 639

Zavlin, V. E., \& Pavlov, G. G. 2004, ApJ, 616, 452

Zharikov, S., Shibanov, Yu., Koptsevich, A., et al. 2002, A\&A, 394, 633

Zharikov, S., Shibanov, Yu., Mennickent R., et al. 2004, A\&A, 417, 1017

Zharikov, S., Shibanov, Yu., \& Komarova, V. 2006, AdSpR, 37, 1979

Zharikov, S., Shibanov, Yu., Mennickent R., \& Komarova, V. 2008, A\&A, 479, 793

Wagner, S. J., \& Seifert, W. 2000, Pulsar Astronomy - 2000 and Beyond, Proceedings of the 177th Colloquium of the IAU held in Bonn, Germany, 30 August-3 September 1999, ed. M. Kramer, N. Wex, \& N. Wielebinski, ASP Conf. Ser., 202, 315

Winkler, P. F., \& Long, K. S. 2006, AJ, 132, 360 